\title{
Extracción y morfología del embrión de Bursera bipinnata (Burseraceae)
}

\section{Extraction and embryo morphology of Bursera bipinnata (Burseraceae)}

\author{
María Felix Ramos-Ordoñez,3, María del Coro Arizmendi', Valeria Flores-Enríquez² y Judith Márquez-Guzmán²
}

1 Universidad Nacional Autónoma de México, Facultad de Estudios Superiores Iztacala, UBIPRO, Laboratorio de Ecología, Avenida de los Barrios núm. 1, Los Reyes Ixtacala, 54090 Tlalnepantla, Estado de México, México.

2 Universidad Nacional Autónoma de México, Facultad de Ciencias, Laboratorio de Desarrollo en Plantas, Avenida Universidad 3000, Coyoacán, Ciudad de México, México.

3 Autor para la correspondencia: rmafyx@yahoo.com.mx

\section{Citar como:}

Ramos-Ordoñez, M., F., M. C. Arizmendi, V. Flores-Enríquez y J. Márquez-Guzmán. 2016. Extracción y morfología del embrión de Bursera bipinnata (Burseraceae). Acta Botanica Mexicana 117: 27-35.

Recibido: 15 de junio de 2015 Revisado: 18 de mayo de 2016 Aceptado: 19 de agosto de 2016

\section{Resumen:}

Antecedentes y Objetivos: El entendimiento de la biología reproductiva de las plantas, desde la formación de la semilla hasta su establecimiento, permite el desarrollo de diferentes estrategias de conservación. En el género Bursera, cuyo principal centro de diversificación es México, la diáspora es un pireno formado por un endocarpo fuertemente lignificado que encierra a la semilla. Las características anatómicas del pireno y la partenocarpia son los principales factores que han obstaculizado el estudio de la embriogénesis y el desarrollo de técnicas de propagación. En este trabajo se describe la morfología del embrión de Bursera bipinnata extraído mediante una técnica que no altera la estructura o la composición química de los tejidos.

Métodos: Durante el inicio de la etapa de dispersión 2014, se colectaron frutos inmaduros y pirenos con pseudoarilo expuesto, los frutos inmaduros se fijaron en FAA. Se utilizó un micromotor con pieza de mano de baja velocidad. En los frutos inmaduros se utilizó un fresón cónico para desgastar las valvas del fruto. Para abrir los pirenos se utilizó un disco de diamante. Una vez abierto el endocarpo, los embriones se extrajeron y se fijaron.

Resultados clave: El patrón de desarrollo coincide con otras especies modelo de angiospermas. En los frutos inmaduros se encontraron las fases globular, acorazonada, torpedo y cotiledonar, ésta última en etapas iniciales. En la fase de torpedo los cotiledones se ramifican en tres lóbulos y se empalman, a medida que crecen se pliegan sobre si mismos encerrando el eje embrionario; sin embargo, el meristemo radicular siempre queda expuesto. El embrión se clasificó como embrión axial foliado contortuplicado.

Conclusiones: La técnica de extracción es rápida, de bajo costo y no invasiva para los tejidos, teniendo un uso potencial en el análisis detallado de la embriogénesis del género Bursera, el cultivo de tejidos y la conservación ex situ.

Palabras clave: Burseraceae, cotiledón, embriogénesis, pireno, tecnica de extracción, semilla.

\section{ABSTRACT:}

Background and Aims: The understanding of the reproductive biology of plants, from the formation of the seed to its establishment, allows the development of different conservation strategies. In the genus Bursera, whose main center of diversification is Mexico, the diaspore is a pyrene formed by a strongly lignified endocarp enclosing the seed. The anatomical characteristics of the pyrene and the parthenocarpy are the main factors that have hindered the study of embryogenesis and the development of propagation techniques. In this paper we describe the morphology of the Bursera bipinnata embryo, extracted by a technique that does not alter the structure or the chemical composition of tissues.

Methods: During the beginning of the dispersal stage 2014, we collected unripe fruits and pyrenes with exposed pseudaril. Unripe fruits were fixed in FAA. A low speed micromotor with handpiece was used. A conical burr was used in unripe fruits to scabble the fruit valves. To open the pyrenes a diamond disc was used. After opening the endocarp, embryos were removed and fixed.

Key results: The pattern of development coincides with other model species of angiosperms. In immature fruits globular, heart-shaped, torpedo and cotyledonary stages were observed, the latter in early stages. During the torpedo stage, cotyledons branch into three lobes and are spliced, as they grow, they are folded upon themselves, and cover the embryonic axis; however, the radicular meristem is always exposed.

Conclusions: The extraction technique is rapid, inexpensive and non-destructive to tissues, having a potential use in the detailed analysis of embryogenesis in the genus Bursera, tissue culture and ex situ conservation.

Key words: Burseraceae, cotyledon, embryogenesis, extraction technique, pyrene, seed. 


\section{INTRODUCCIÓN}

La semilla es una etapa clave del ciclo de vida de las angiospermas, ya que representa a la nueva generación esporofítica (Márquez-Guzmán et al., 2012), mientras que su dispersión es la oportunidad de colonizar nuevos sitios y permite a los nuevos genotipos encontrar las condiciones ambientales óptimas para su desarrollo (Fahn y Werker, 1972; Nathan y Muller-Landau, 2000). Ambos procesos juegan un papel fundamental en la dinámica y regeneración de las comunidades vegetales, así como en el uso de especies nativas en programas de conservación, reforestación, manejo y restauración de los ecosistemas, por lo que su estudio, desde diversos enfoques disciplinarios, reviste enorme importancia.

Los copales y cuajiotes del género Bursera Jacq. ex L. (Burseraceae) comprenden 107 especies de árboles y arbustos resinosos, distribuidos en el bosque tropical estacionalmente seco, desde el norte de México hasta el norte de Sur América, excepto por B. tonkinensis Guillaumin que se encuentra en el norte de Vietnam, siendo México el principal centro de diversidad (De-Nova et al., 2012). Por su riqueza, abundancia y cantidad de endemismos, es uno de los géneros más importantes del Hotspot Mesoamericano (Myers et al., 2000); sin embargo, se encuentra sometido a una gran presión debido a la reducción del hábitat (Meave et al., 2012). Particularmente algunas especies están sometidas a la explotación no sustentable para uso maderable, elaboración de artesanías, medicina tradicional, ornato, y extracción de resinas con fines ceremoniales (Ramos-Ordoñez, 2009). Por su amplia distribución y/o importancia económica, varias especies, como B. bipinnata (DC.) Engl., B. copallifera (DC.) Bullock, B. glabrifolia (Kunth) Engl., B. linanoe (La Llave) Rzedowski, Calderón y Medina, y B. simaruba (L.) Sarg., Gard. y For., son objeto de diversos estudios con fines de restauración (Vázquez-Yanes et al., 1999; Bonfil-Sanders et al., 2008; Orantes-García et al., 2013; GonzálezOrozco, 2014; Vázquez-Cisneros, 2014). Sin embargo, la propagación de las especies ha sido fuertemente limitada por la partenocarpia o producción de frutos sin semilla, un fenómeno común y natural en el género (Ramos-Ordoñez et al., 2012), así como por la falta de conocimiento de la diáspora y de la biología reproductiva de las especies (Bonfil-Sanders et al., 2008; Orantes-García et al., 2013).

En muchas especies de plantas, la semilla se separa de la planta madre acompañada de otros tejidos $u$ órganos provenientes del fruto o de la flor, en cuyo caso la unidad de dispersión se denomina diáspora (Fahn y Werker, 1972; Vittoz y Engler, 2007; Márquez-Guzmán et al., 2012; Baskin y Baskin, 2014). Cuando la semilla de Bursera madura, el exocarpo y gran parte del mesocarpo se desprenden en valvas, dejando al descubierto la diáspora; la semilla permanece cubierta por el endocarpo fuertemente lignificado, a esta estructura se le denomina pireno (Daly et al., 2011; Ramos-Ordoñez et al., 2012). Éste está cubierto parcial o completamente por un pseudoarilo brillantemente coloreado y rico en lípidos, que funciona como atrayente y recompensa para los dispersores (Ramos-Ordoñez et al., 2012). El endocarpo duro dificulta la observación de la semilla y del embrión, y es la causa principal de que la embriogénesis de las semillas de Bursera no se haya descrito hasta la fecha.

El desarrollo embrionario describe el periodo durante el cual el cigoto se transforma en un embrión maduro. Durante la embriogénesis (sensu stricto) ocurre la morfogénesis. Durante la fase de proembrión ocurre la primera división del cigoto, que incluye la formación del suspensor y de la primera célula del embrión propiamente dicho. Posteriormente ésta célula se divide formando al embrión globular; durante la fase de transición éste da lugar a un embrión acorazonado en el que se establece la polaridad raíz-tallo del eje embrionario, se forman órganos y tejidos, se forman y desarrollan los meristemos caulinar y radicular, y se inicia la formación de los cotiledones. Una vez que se detiene la morfogénesis, domina el crecimiento celular, el eje embrionario y los cotiledones se alargan, se sintetizan y acumulan sustancias de reserva, se adquiere tolerancia a la desecación, se inhibe la germinación precoz, y en algunas especies se desarrolla la latencia vinculada a la desecación de la semilla (Goldberg et al., 1994; Dodeman et al., 1997; Braybrook y Harada, 2008). Estos cambios proporcionan las características 
embrionarias necesarias para formar semillas aptas para la dispersión de la progenie. En este contexto, la extracción de la semilla y/o el embrión intactos conlleva varias ventajas para el conocimiento de la biología reproductiva de Bursera, incrementando las posibilidades de uso de estas especies en planes de restauración ecológica, silvicultura o plantaciones industriales, por citar algunos. En este trabajo se describe la morfología del embrión de Bursera bipinnata, extraído del pireno, mediante una técnica rápida y de bajo costo que permite su extracción sin causar daño a los tejidos ni alterar su composición química.

\section{MATERIALES Y MÉTOdOS}

Bursera bipinnata es una de las especies del género Bursera con más amplia distribución, desde Durango, México hasta Honduras y El Salvador. En México es un elemento moderadamente frecuente del bosque tropical seco, se le puede encontrar en los márgenes de encinares y pinares adyacentes, en altitudes de 800 a 1600 m (Rzedowski et al., 2004). La recolecta de material biológico se hizo durante agosto de 2014, en la zona arqueológica de Xochicalco y la barranca del Río Tembembe, municipio de San Agustín Tetlama, Morelos, México ( $18^{\circ} 50^{\prime} \mathrm{N}$ y 99¹9'O). De acuerdo con la fenología de la fructificación y las características morfológicas del fruto y del pireno descritas por Ramos-Ordoñez et al. (2012), se seleccionaron al azar cuatro árboles. De cada uno se recolectaron 50 frutos inmaduros y 50 pirenos con pseudoarilo expuesto. Los frutos inmaduros se fijaron en FAA (formol, ácido acético, etanol 96\% y agua 1:0.5:5:3.5), y los pirenos se almacenaron en bolsa de papel. Adicionalmente se colectaron 20 frutos inmaduros por árbol y se almacenaron, sin fijar, en bolsa de papel. Las muestras se llevaron al laboratorio de Desarrollo en Plantas, Facultad de Ciencias, UNAM y se almacenaron en oscuridad y condiciones ambiente. Se tomaron muestras al azar de cada uno de los tres grupos recolectados para extraer los embriones $(n=20$ de cada grupo), tanto en tejido fijado (frutos inmaduros) como en tejido fresco (frutos inmaduros sin fijar y pirenos con pseudoarilo expuesto). Previo a la extracción de la semilla se midió el largo y ancho de los frutos inmaduros y los pirenos. Después de la extracción de las semillas se midió el grosor del endocarpo (en todos los casos se presenta el valor promedio acompañado de la desviación estándar).

Para la extracción de las semillas se utilizó un micromotor (MDC Dental ${ }^{\circledR}$ Escort-III, Múnich, Alemania) con pieza de mano de baja velocidad; los modelos de baja velocidad son convenientes por su bajo costo y son de uso común entre odontólogos y protesistas. También se utilizó un fresón cónico de punta redonda (NTI ${ }^{\circledR} \mathrm{HF} 079 \mathrm{CE}-045$, Múnich, Alemania); la punta redonda facilita la creación de surcos con ángulos redondeados, mientras que la forma cónica proporciona paredes inclinadas y un fácil desgaste de la superficie en comparación con una forma cilíndrica. Finalmente se utilizó un disco diamantado $\left(\mathrm{NTI}^{\circledR}\right.$ 358.514.220, Múnich, Alemania); este tipo de disco se utiliza para cortar aleaciones y cerámicas para prótesis dentales. Es importante considerar que los instrumentos de $4 \mathrm{~mm}$ de diámetro deben utilizarse a una velocidad máxima de 20000 r.p.m. (NTI, 2011), en este estudio la velocidad utilizada no excedió las 7000 r.p.m.

Los frutos inmaduros previamente fijados se lavaron con agua corriente para retirar el fijador y se deshidrataron en una serie de alcoholes etílicos graduales hasta etanol 70\% (López et al., 2005). El resto del material se procesó directamente. Para exponer el pireno en los frutos inmaduros, primero se retiró el exocarpo y el mesocarpo (valvas del fruto); estos tejidos son relativamente blandos y se desgastan fácilmente con el fresón cónico de punta redonda (Fig. 1A). En los pirenos con pseudoarilo expuesto, las valvas se caen de manera natural (Fig. 1B), el pseudoarilo se retiró manualmente.

Para abrir el pireno y extraer la semilla se utilizó el disco de diamante. Bajo el estereomicroscopio (Zeiss Stemi CV4, Oberkochen, Alemania), se dibujaron con el disco dos cortes longitudinales simulando una "tapa" sobre la superficie del pireno (Fig. 1B y 1C). Una vez realizados los cortes guía, se continuó desgastando el endocarpo con el disco, al alcanzar el espacio de la cavidad seminal, u observar un cambio de coloración en el endocarpo, se retiró la "tapa" formada con unas pinzas de disección (Fig. 1D). Una vez que se observó el tamaño de la semilla, se 
retiró el endocarpo tanto como fue necesario usando el disco de diamante, hasta que se consideró que se podía extraer la semilla. Cuando se encontró la semilla en etapa temprana del desarrollo, primero fue necesario despegarla del endocarpo moviéndola ligeramente con una aguja de disección. Una vez suelta, se retiró suavemente con ayuda de unas pinzas o un pincel (Fig. 1E). En etapas tardías del desarrollo, la semilla se extrajo jalando cuidadosamente una pequeña porción de la cubierta seminal con pinzas de punta fina (Fig. 1F). La testa es un tejido de tipo parenquimático por lo que es muy fácil romperla; sin embargo, es posible extraer la semilla completa sin daños.

En etapas tempranas de desarrollo, las semillas miden 1-2 mm de largo y el embrión se extrajo rompiendo la testa, por el lado contrario al micrópilo, con ayuda de dos agujas de disección o alfileres entomológicos. Debido a su pequeño tamaño, el embrión siempre se conservó embebido en el endospermo o unido a una porción de la testa. En etapas avanzadas de desarrollo de la semilla (2-4 $\mathrm{mm}$ ), el embrión se extrajo rompiendo la testa y retirándola en segmentos con unas pinzas de punta fina. En esta etapa es fácil ver el embrión con ayuda de la luz inferior del estereomicroscopio. Los embriones se almacenaron en FAA durante dos días, posteriormente se lavaron y se deshidrataron hasta alcohol etílico $70 \%$ para la toma de fotografías.

Se utilizaron cinco embriones en etapas tempranas de desarrollo y se procesaron anatómicamente para su presentación en este trabajo. Dichos embriones se incluyeron en LR-White, se cortaron con cuchillas de vidrio en ultramicrotomo (RMC-MT 990, Arizona, EUA), a $1.5 \mu$ y se tiñeron con azul de toluidina, de acuerdo con el manual de López et al., (2005). Las etapas avanzadas de desarrollo se presentan sin procesamiento posterior a la extracción. La clasificación del embrión se realizó de acuerdo con Gunn (1972). Se realizó un registro fotográfi-

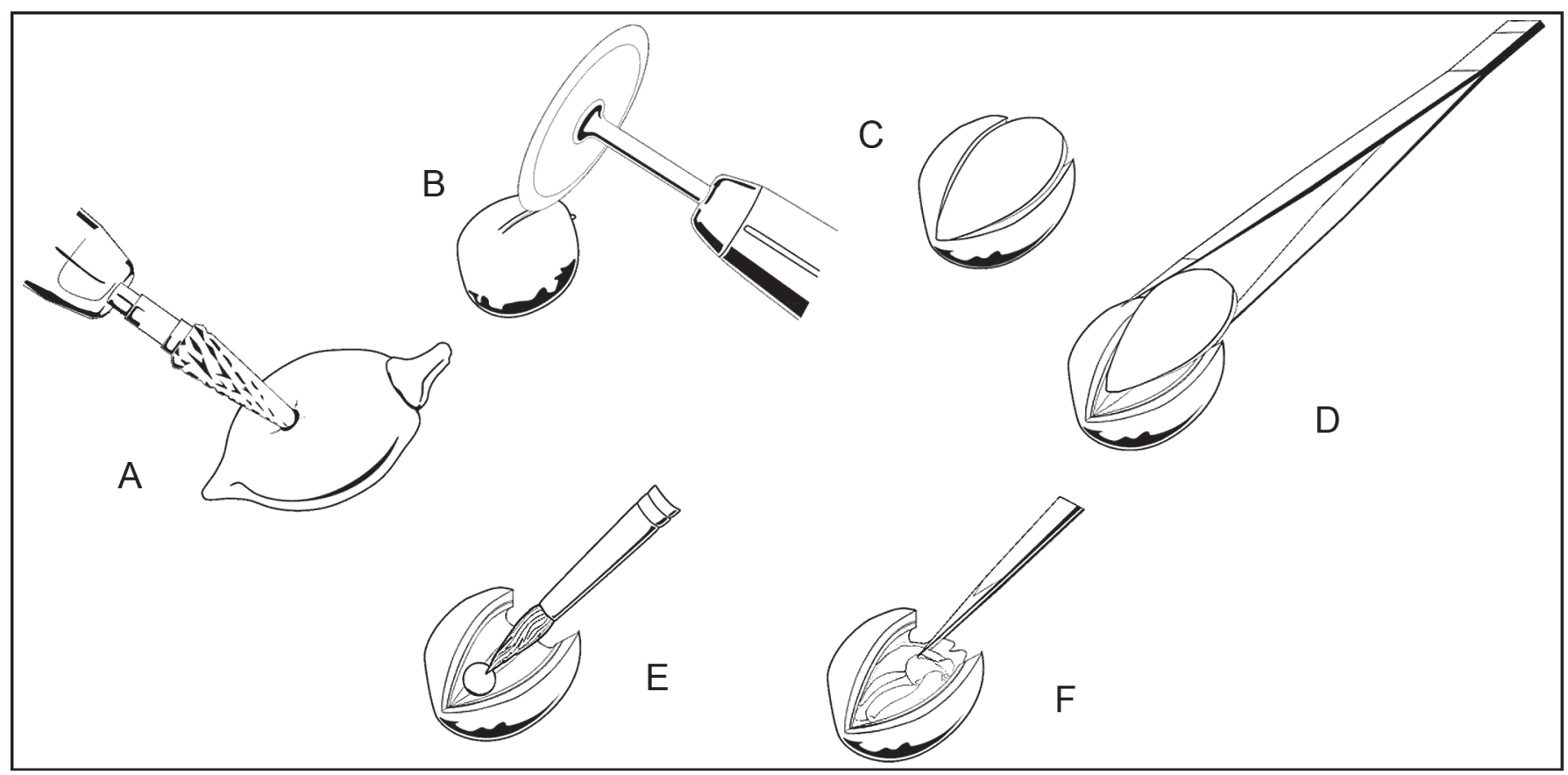

Figura 1: Técnica de extracción de la semilla de Bursera bipinnata (DC.) Engl. A. desgaste del exocarpo y mesocarpo con fresón cónico de punta redondeada; B. corte del endocarpo con disco de diamante; C. se realizan dos líneas de corte longitudinales con el disco de diamante para formar una tapa; D. la tapa se retira para determinar el estado de desarrollo de la semilla y continuar desgastando el endocarpo si es necesario; E. en etapas tempranas de desarrollo, la semilla no llena la cavidad seminal y puede extraerse con pincel; F. en etapas avanzadas de desarrollo, la semilla se extrae jalando con la pinza de disección una porción de la testa. 
co usando un microscopio óptico (Olympus Provis AX70, Tokio, Japón), y estereomicroscopio (Zeiss, Oberkochen, Alemania).

\section{Resultados}

Los frutos de Bursera bipinnata son semiesféricos y miden $6.68 \pm 0.57 \mathrm{~mm}$ de largo por $5.85 \pm 0.35 \mathrm{~mm}$ de ancho. El ovario es bilocular, en cada lóculo se forman dos óvulos. Durante el desarrollo, uno de los lóculos se oblitera y sus óvulos abortan, en el segundo lóculo, solo uno de los óvulos aborta y el segundo permanece viable, pero detiene su crecimiento durante uno a tres meses (Fig. 2A). Los pirenos son ligeramente aplanados dorsoventralmente, miden $4.84 \pm 0.71 \mathrm{~mm}$ de largo por $3.76 \pm 0.29 \mathrm{~mm}$ de ancho y $2.81 \pm 0.26 \mathrm{~mm}$ en la zona dorsoventral media (Fig. 2B), las valvas y el pseudoarilo miden en promedio $2 \mathrm{~mm}$ de espesor. El endocarpo mide $0.43 \pm 0.12 \mathrm{~mm}$ de grosor, excepto en la zona donde se oblitera el lóculo que es más gruesa $1.22 \pm 0.17 \mathrm{~mm}$. Se encontraron frutos partenocárpicos $(n=5)$, en los que el mesocarpo y endocarpo invadieron el lóculo obliterando el óvulo.

Cuando las semillas inmaduras detienen su crecimiento, miden apenas $1 \mathrm{~mm}$ (longitud micrópilo - calaza), son semiesféricas y se encuentran unidas a la pared del fruto por medio del funículo (Fig. 3A). Las etapas embrionarias encontradas en este periodo son la globular y la acorazonada. Cuando la semilla mide de 1 a $3 \mathrm{~mm}$ de longitud (Fig. 3B), en la zona del micrópilo, se puede observar el embrión en etapa de torpedo o cotiledonar. En la etapa de torpedo, cada cotiledón se trifurca y toma una posición en ángulo recto con respecto al eje embrionario, en esta etapa, la longitud de los cotiledones siempre es menor a la del eje embrionario (Fig. 3C).

En la etapa cotiledonar se observa principalmente el crecimiento y acomodo de los cotiledones trilobados. Ambos cotiledones se empalman dando la apariencia de ser uno solo, el meristemo apical queda oculto en la base de los cotiledones; a trasluz se puede observar la ramificación de los haces vasculares, en esta etapa los lóbulos de los cotiledones tienen aproximadamente la misma longitud del eje embrionario (Fig. 3D). A medida que los cotiledones se alargan, tocan las paredes del endocarpo y se curvan hacia adentro (Fig. 3E); los cotiledones continúan alargándose y ensanchándose hasta tocarse a nivel del meristemo apical, en una vista dorsal se puede apreciar la primera torsión (Fig. 3F). Cuando los cotiledones se en-
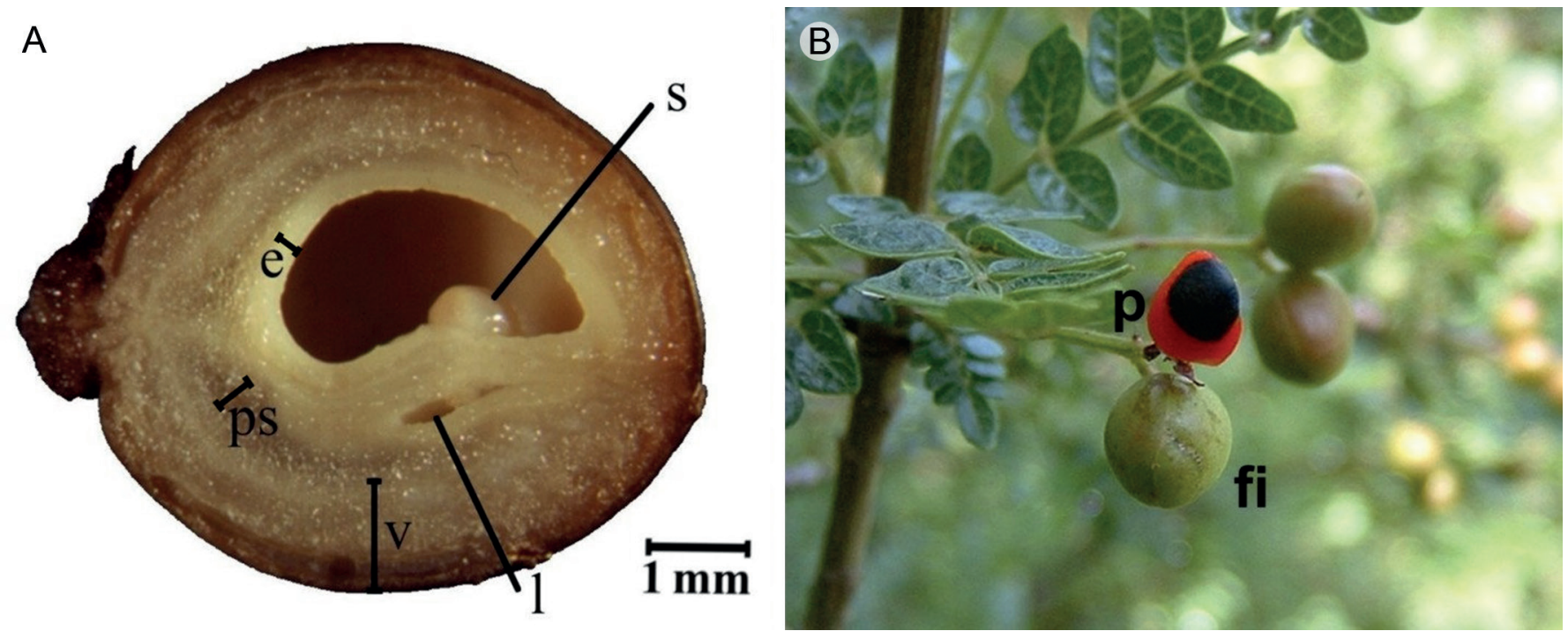

Figura 2: Bursera bipinnata (DC.) Engl. A. corte longitudinal del fruto inmaduro, e. endocarpo, 1. lóculo obliterado, ps. pseudoarilo, s. semilla en estado temprano de desarrollo dentro de la cavidad seminal, v. valva; B. pireno con el pseudoarilo expuesto y frutos inmaduros, fi. fruto inmaduro, p. pireno. 
cuentran en el centro se curvan hacia afuera sobre sí mismos, esto se repite dos (Fig. 3G) a tres veces (Fig. 3H), el ensanchamiento de los cotiledones termina encerrando el eje embrionario (Fig. 3I). Finalmente, los cotiledones se compactan en torno al embrión de manera que solo dejan ver el meristemo radicular (Fig. 3J). La testa (Fig. 3K) es una capa delgada y traslucida excepto en la zona del funículo que es más obscura (Fig. 3L) y prácticamente no se observa endospermo (Fig. 3M). Cuando el embrión se ha desarrollado por completo puede verse alargado, o en forma de corazón (Figs. 3K y 3N), o semiesférico. Esto dependerá del espacio disponible en el lóculo; sin embargo, en todos los casos el embrión se puede clasificar como embrión axial foliado contortuplicado. Los embriones globular y acorazonado fueron demasiado pequeños para la obtención de microfotografías; sin embargo, la técnica

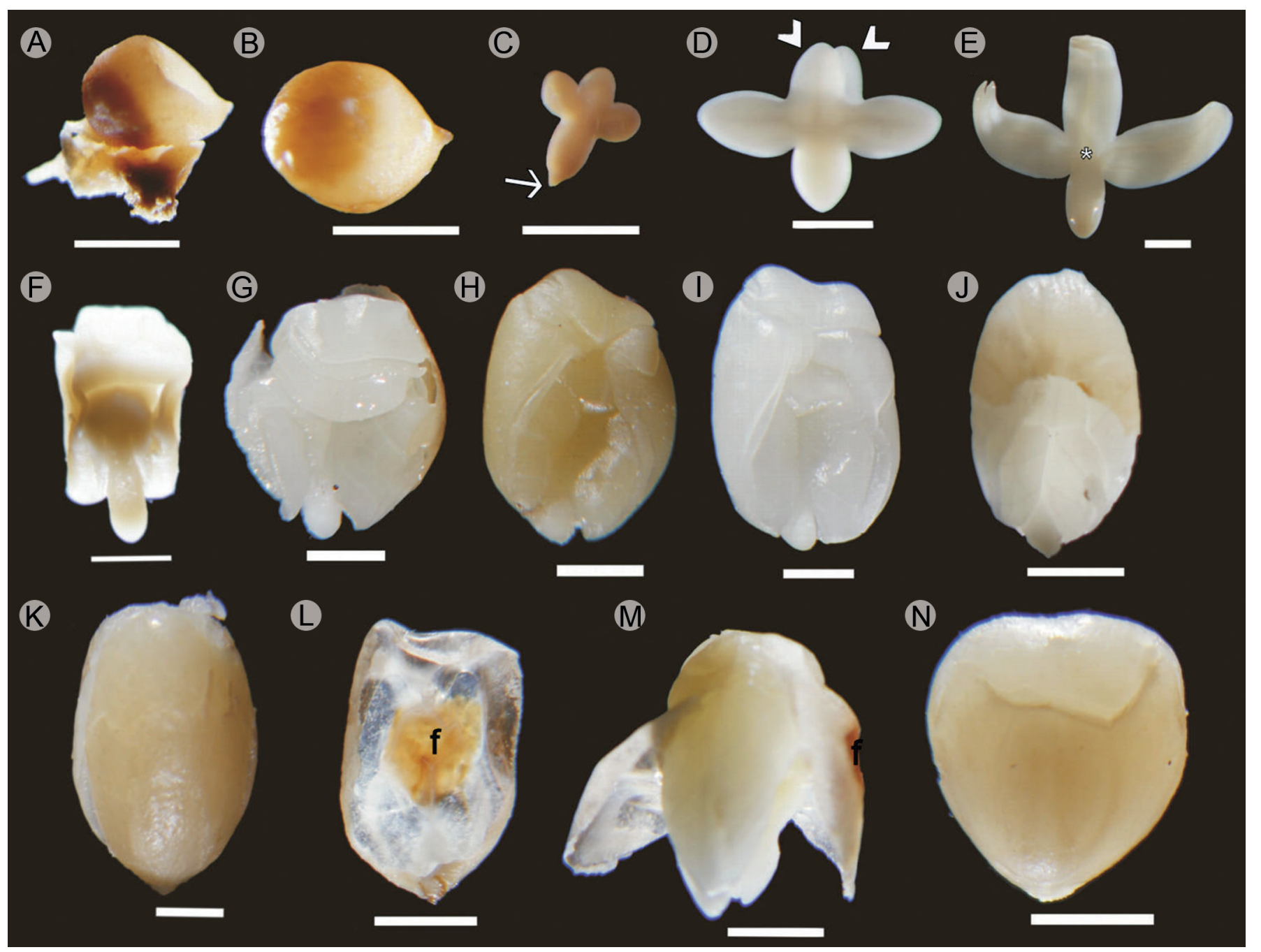

Figura 3: Etapas de desarrollo del embrión de Bursera bipinnata (DC.) Engl. A. semilla con embrión en estado de suspensión unida a una porción del endocarpo; B. semilla inmadura que ha reiniciado el desarrollo; C. embrión en etapa de torpedo, aún se observa el suspensor (flecha); D. inicio de la etapa cotiledonar, se señalan los lóbulos apicales de ambos cotiledones (cabezas de flecha); E. embrión cotiledonar iniciando la primera torsión, se señala a que nivel se encuentra el meristemo apical (*); F. vista dorsal del embrión cotiledonar al finalizar la primera torsión, los cotiledones se ensanchan; G. embrión con dos torsiones; H. embrión con tres torsiones; I. el ensanchamiento de los cotiledones encierra el eje embrionario; J. embrión desarrollado, la porción media superior muestra la testa, la porción media inferior permite ver el meristemo radicular libre de cotiledones; K. semilla en vista dorsal; L. porción ventral de la testa de k, se muestra el funículo (f); M. semilla con la testa disectada, se aprecia la falta de endospermo y el grosor de la testa; N. semilla madura. Macrofotografías tomadas con estereomicroscopio. Barra=1 mm. 
de extracción utilizada permitió procesar las muestras por lo que se hicieron cortes histológicos (Fig. 4).

\section{DISCUSIÓN}

Las etapas embrionarias globular, acorazonada, de torpedo y cotiledonar en este trabajo coinciden con el modelo propuesto para Arabidopsis thaliana (L.) Heynh. (Dodeman et al., 1997) y otras especies modelo de angiospermas (Márquez-Guzmán et al., 2012). No se observaron etapas embrionarias anteriores a la globular, como sucede en Dacryodes edulis (G. Don) H.J. Lam (Burseraceae), en la cual la primera división del cigoto ocurre ocho horas después de la polinización; sin embargo, la semilla entra en un aparente estado de latencia y el desarrollo del embrión es visible hasta después de dos meses (Kengue, 2002).

Debido a que la embriogénesis ocurre en el interior de la semilla, su observación generalmente se realiza mediante estudios anatómicos (Dodeman et al., 1997). Sin embargo, los cortes son particularmente difíciles cuando, entre otras circunstancias, la cubierta de la semilla es muy dura, o cuando el método de ablandamiento daña el tejido blando del embrión, o cuando es difícil orientar correctamente la semilla para observar el embrión. La técnica aquí descrita es rápida, económica y no invasiva para los tejidos que se desean obtener. A partir de ella fue posible conocer parte de la ontogenia de la semilla, desconocida para Bursera, y se podrían obtener tejidos seminales para la propagación in vitro con fines comerciales o de conservación. En la familia Burseraceae, la inducción de embriones somáticos a partir de explantes de embriones cigóticos únicamente se ha realizado en Commiphora wightii (Arn.) Bhandari, y requiere de la selección de embriones cigóticos en etapas específicas de desarrollo para obtener un máximo rendimiento (Kumar et al., 2006; Kumar y Nadgauda, 2015). En Bursera linanoe la propagación in vitro inicia con la obtención de los cotiledones y hojas en expansión; sin embargo, los protocolos de estudio aún se encuentran en desarrollo (González-Orozco, 2014; Vázquez-Cisneros, 2014), por lo que la aplicación de esta técnica resultaría en un ahorro sustancial de tiempo para la obtención de explantes.

Dado que la partenocarpia es un factor que reduce la cantidad de pirenos con semilla, en algunos casos hasta

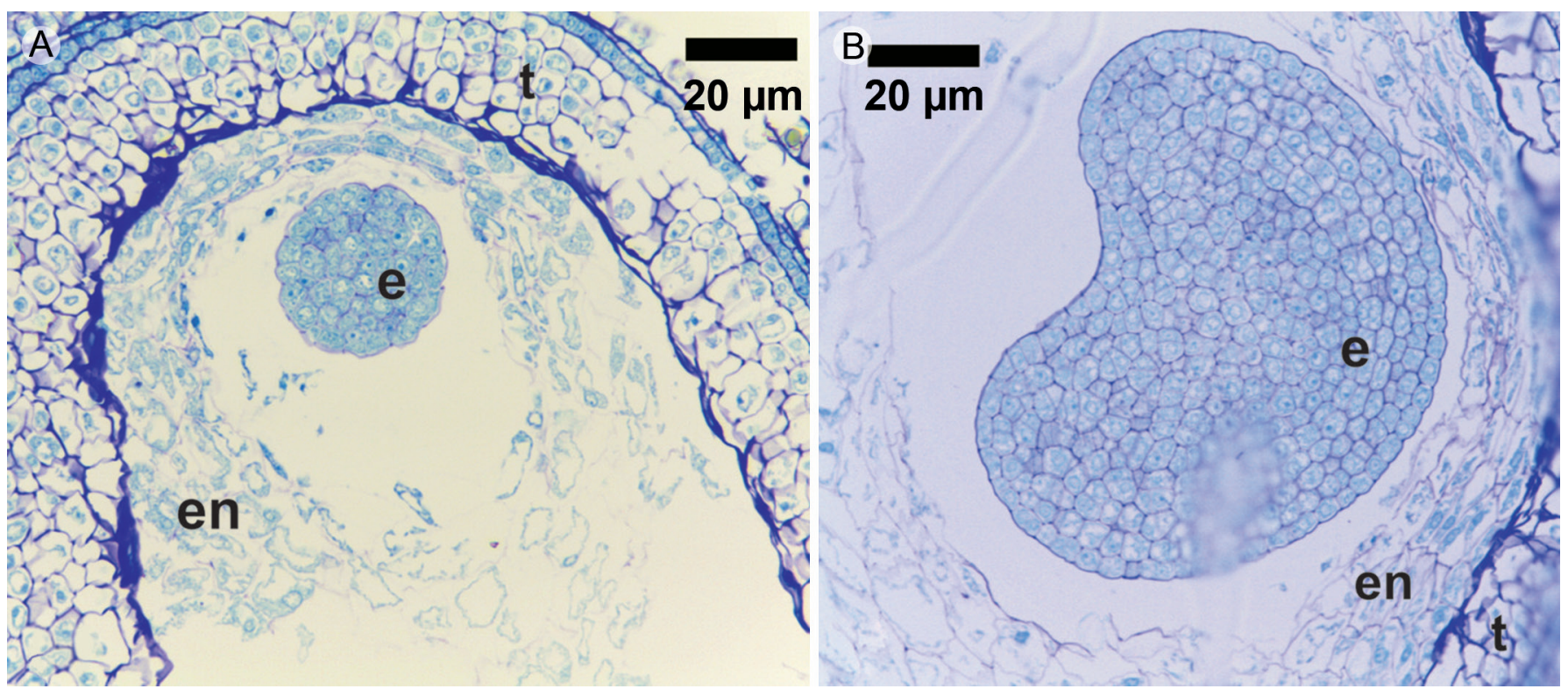

Figura 4: Bursera bipinnata (DC.) Engl. A. etapa globular; B. etapa acorazonada. e. embrión; en. endospermo; t. testa. Microfotografías tomadas en microscopía de campo claro. Barra=20 $\mu \mathrm{m}$. 
$50 \%$ de la cosecha es partenocárpica (Ramos-Ordoñez et al., 2012). La conservación ex situ de Bursera en bancos de semillas presenta varios problemas, derivados del desconocimiento del contenido de los pirenos, ya que las recolectas se hacen al azar y en diferentes tiempos de la fenología de maduración del fruto, incluso cuando el óvulo se encuentra en reposo. Las pruebas de germinación que se realizan periódicamente en la colección arrojan porcentajes de germinación nulos o muy bajos, asumiendo que se trata de frutos partenocárpicos (I. Rodríguez, com. pers.). En este trabajo los frutos de B. bipinnata formados en junio de 2014 (M. F. Ramos Ordoñez, obs. pers.) comenzaron a madurar asincrónicamente en agosto cuando se hizo la recolecta, todos los frutos con valvas presentaron embriones inmaduros o eran frutos partenocárpicos. Únicamente los pirenos con pseudoarilo expuesto presentaron un embrión con desarrollo completo, es decir, llenaba la cavidad seminal, no presentaba malformaciones y la semilla carecía de endospermo. Esta información muestra la importancia de reconocer la fenología de la maduración de la semilla en el momento de recolectar frutos o pirenos para su almacenamiento, por lo que la técnica aquí presentada permitirá establecer una correspondencia entre patrones morfológicos externos de los pirenos y la etapa de desarrollo del embrión en otras especies de Bursera, de manera que se utilicen pirenos que aseguren altos porcentajes de germinación durante las pruebas de viabilidad de las colecciones.

El fruto de B. bipinnata muestra patrones estructurales y fenológicos muy similares a los de 15 especies más del género, dichos patrones se han establecido a nivel del complejo Commiphora-Bursera (Ramos-Ordoñez et al., 2012; 2013), sugiriendo que la técnica puede aplicarse en Commiphora Jacq. e incluso en otras burseráceas de importancia comercial, cultural y ecológica (Daly et al., 2011).

\section{AgRADECIMIENTOS}

El presente trabajo contó con el apoyo del proyecto PAPIIT IN217511-3 de MC Arizmendi; el Programa de Apoyos Complementarios para la Consolidación Institucional de Grupos de Investigación CONACyT (206096) de MF
Ramos-Ordoñez, así como el presupuesto interno del Laboratorio de Desarrollo en Plantas. Ana Isabel Bieler asistió en la toma de fotografías, Espinosa-Sánchez M. asistió en las técnicas anatómicas, Machuca C. asistió en la colecta de campo y elaboró la Figura 1.

\section{LITERATURA CITADA}

Baskin, C. C. y J. M. Baskin. 2014. Seeds: Ecology, biogeography, and evolution of dormancy and germination. 2nd ed. Academic Press/Elsevier. London, UK. 1601 pp.

Bonfil-Sanders, C., I. Cajero-Lázaro y R. Y. Evans. 2008. Germinación de semillas de seis especies de Bursera del centro de México. Agrociencia 42: 827-834.

Braybrook, S. A. y J. J. Harada. 2008. LECs go crazy in embryo development. Trends in Plants Science 13: 64-630.

Daly, D. C., M. C. Martínez-Habibe, A. Weeks y M. M. Harley. 2011. Burseraceae. In: Kubitzki, K. (ed.). Flowering plants, Eudicots, Sapindales, Cucurbitales, Myrtaceae, The families and genera of vascular plants. Vol X. Springer-Verlag. New York, USA. pp. 76-104.

De-Nova, J. A., R. Medina, J. C. Montero, A. Weeks, J. A. Rosell, M. E. Olson, L. E. Eguiarte, y S. Magallón. 2012. Insights into the historical construction of species-rich Mesoamerican seasonally dry tropical forests: the diversification of Bursera (Burseraceae, Sapindales). New Phytologist 193: 276-287. DOI: http://dx.doi.org/10.1111/ j.1469-8137.2011.03909.x.

Dodeman, V. L., G. Ducreux y M. Kreis. 1997. Zygotic embryogenesis versus somatic embryogenesis. Journal of Experimental Botany 48: 1493-1509.

Fahn, A. y E. Werker. 1972. Anatomical mechanisms of seed dispersal. In: Kozlowski, T. T. (ed.). Seed biology. Physiological ecology. A series of monographs, texts and treatises. Vol I, Importance, development, and germination. Academic Press. New York, USA. pp. 151-221.

Goldberg, R. B., G. de Paiva, y R. Yadegari. 1994. Plant Embryogenesis: Zygote to seed. Science, New Series 266: 605-614. DOI: http://dx.doi.org/10.1126/science.266.5185.605

González-Orozco, M. M. 2014. Maduración y germinación de embriones somáticos de lináloe In vitro y Ex vitro. Tesis 
de maestría. Colegio de Postgraduados. Estado de México, México. 159 pp.

Gunn, C. R. 1972. Seed collecting and identification. In: Kozlowski, T. T. (ed.). Seed biology. Physiological ecology. A series of monographs, texts and treatises. Vol III. Insects, and seed collection, storage, testing, and certification. Academic Press. New York, USA. pp. 55-143.

Kengue, J. 2002. Fruits of the future 3. Safou: Dacryodes edulis G. Don. International Centre for Underutilized Crops. Southampton, UK. 147 pp.

Kumar, S., M. Mathur, A. K. Jain y K. G. Ramawat. 2006. Somatic embryo proliferation in Commiphora wightii and evidence for guggulsterone production in culture. Indian Journal of Biotechnology 5: 217-222.

Kumar, S. y R. Nadgauda. 2015. Control of morphological aberrations in somatic embryogenesis of Commiphora wightii (Arnott) Bhandari (Family Burseraceae) through secondary somatic embryogenesis. Proceedings of the National Academy of Sciences, India Section B: Biological Sciences 85: 281-290.

López, C. M. L., J. Márquez y G. Munguía. 2005. Técnicas para el estudio del desarrollo en angiospermas. $2^{\mathrm{a}}$. ed. Universidad Nacional Autónoma de México. D.F., México. 116 pp.

Márquez-Guzmán, J., M. Collazo, M. Martínez, A. Orozco y S. Vázquez. 2012. Biología de angiospermas. Facultad de Ciencias, Universidad Nacional Autónoma de México-Coordinación de Servicios Editoriales. D.F., México. $632 \mathrm{pp}$.

Meave, J. A., A. Romero-Romero, H. Salas-Morales, A. PérezGarcía y A. Gallardo-Cruz. 2012. Diversidad, amenazas y oportunidades para la conservación del bosque tropical caducifolio en el estado de Oaxaca, México. Ecosistemas 21: 85-100.

Myers, N., R. A. Mittermeier, G. Mittermeier, A. B. Da Fonseca y J. Kent. 2000. Biodiversity hotspots for conservation priorities. Nature 403: 853-858.

Nathan, R. y C. Muller-Landau. 2000. Spatial patterns of seed dispersal, their determinants and consequences for recruitment. Trends in Ecology and Evolution 15: 278-285.
NTI. 2011. Catalogue Rotary Dental Instruments Laboratory. New Technology Instruments. Kahla, Germany. 164 pp.

Orantes-García, C., A. Pérez-Farrera, M. Rioja-Paradela y R. Garrido-Ramírez. 2013. Viabilidad y germinación de semillas de tres especies arbóreas nativas de la selva tropical, Chiapas, México. Polibotánica 36: 117-127.

Ramos-Ordoñez, M. F. 2009. Dispersión biótica de semillas y caracterización de los frutos de Bursera morelensis en el Valle de Tehuacán, Puebla. Tesis de Doctorado. Universidad Nacional Autónoma de México. D.F., México. 92 pp.

Ramos-Ordoñez, M. F., M. C. Arizmendi y J. Márquez-Guzmán. 2012. The fruit of Bursera: structure, maturation and parthenocarpy. AoB Plants. DOI: http://dx.doi. org/10.1093/aobpla/pls027

Ramos-Ordoñez, M. F., M. C. Arizmendi, M. Martínez y J. Márquez-Guzmán. 2013. The pseudaril of Bursera and Commiphora, a foretold homology? Revista Mexicana de Biodiversidad 84: 509-520. DOI: http://dx.doi. org/10.7550/rmb.32114

Rzedowski, J., R. Medina y G. Calderón. 2004. Las especies de Bursera (Burseraceae) en la cuenca superior del Río Papaloapan (México). Acta Botanica Mexicana 66: 23-151.

Vázquez-Cisneros, I. 2014. Producción de embriones somáticos de Bursera linanoe (La Llave) Rzed., Calderón \& Medina, en biorreactores de sistema de inmersión temporal. Tesis de maestría. Colegio de Postgraduados. Estado de México, México. 94 pp.

Vázquez-Yanes, C., I. Batis-Muñoz, A. I. Alcocer-Silva, M. Gual-Díaz y C. Sánchez-Dirzo. 1999. Árboles y arbustos potencialmente valiosos para la restauración ecológica y la reforestación. Reporte técnico del proyecto J084. Comisión Nacional para el Conocimiento y Uso de la Biodiversidad - Instituto de Ecología, Universidad Nacional Autónoma de México. D.F., México. pp. 201-204.

Vittoz, P. y R. Engler. 2007. Seed dispersal distances: a topology based on dispersal modes and plant traits. Botanica Helvetica 117: 109-124. 